\title{
COPS6 Gene
}

National Cancer Institute

\section{Source}

National Cancer Institute. COPS6 Gene. NCI Thesaurus. Code C124917.

This gene is involved in signalosome function. 